\title{
Supplementary
}

\section{Biomimetic and Self-Assembled Nanoclusters Targeting $\beta$-catenin for Potent Anticancer Therapy and Enhanced Immunotherapy}

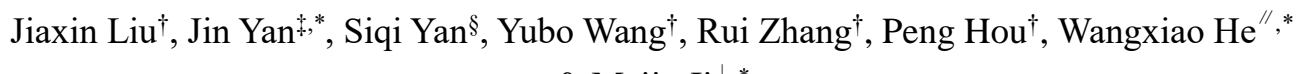
$\&$ Meiju $\mathrm{Ji}^{\perp, *}$

†Key Laboratory for Tumor Precision Medicine of Shaanxi Province and Department of Endocrinology, The First Affiliated Hospital of Xi' an Jiaotong University, Xi' an 710061, China FFrontier Institute of Science and Technology, The Key Laboratory of Biomedical Information Engineering of Ministry of Education, School of Life Science and Technology, Xi'an Jiaotong University, Xi'an 710049, China

§Ophthalmology Department, The First Affiliated Hospital of Xi' an Jiaotong University, Xi'an 710061, China

"Department of Talent Highland, The First Affiliated Hospital of Xi'an Jiaotong University, Xi'an 710061, China.

${ }^{\perp}$ Center for Translational Medicine, The First Affiliated Hospital of Xi'an Jiaotong University, Xi'an 710061, China

\footnotetext{
* Corresponding authors:

*phone: (+86) 13572567052. Email: yanjin19920602@xjtu.edu.cn (J Yan);

* phone: (+86) 15319910808. Email: hewangxiao5366@xjtu.edu.cn (W. He);

*phone: (+86) 18991232538. Email: mjji0409@163.com (M. Ji).
} 


\section{Experimental Section}

\section{General remarks}

All synthetic peptide sources were obtained from CS Bio (Shanghai) Ltd. All other chemicals used in this study were purchased from Sigma-Aldrich unless otherwise specified. Acetonitrile and water (HPLC grade) were purchased from Fisher Scientific Ltd. All products were used as received without further purification.

\section{Synthesis of 2-mercaptoimidazole (MM) modified mercapto polyethylene glycol (PEG)}

MM-NHS was made by conjugating MM to N-succinimidyl 3-maleimidopropionate at molar rate of 1:1 in PBS buffer (pH 7.4). Next, MM-NHS reacted to NH2-PEG-SH (MW 1500 Da) at molar rate of 2:1. The final product MM-PEG-SH was obtained after dialysis to remove the excess MM-NHS.

\section{Synthesis of $\mathrm{H} 1$ peptides}

H1 peptides were synthesized on appropriate resins on an CS bio 336X automated peptide synthesizer using the optimized HBTU activation/DIEA in situ neutralization protocol developed by an HBTU/HOBt protocol for Fmoc-chemistry SPPS. After cleavage and deprotection in a reagent cocktail containing $88 \%$ TFA, $5 \%$ phenol, $5 \% \mathrm{H}_{2} \mathrm{O}$ and $2 \%$ TIPS, crude products were precipitated with cold ether and purified to homogeneity by preparative C18 reversed-phase HPLC. The molecular masses were ascertained by electrospray ionization mass spectrometry (ESI-MS).

\section{Fabrication of $\mathrm{CA}_{\mathrm{cluster}}$}

The as-prepared H1 and MM-PEG-SH were dispersed in PBS (pH 7.4) at molar rate of 4:1. 
Next, 2-fold excess gadolinium chloride $\left(\mathrm{GdCl}_{3}\right)$ were added into the buffer. After a 20-min incubation with stirring at $30^{\circ} \mathrm{C}$, the Gd-H1/PEG was obtained after dialysis to remove the excess $\mathrm{Gd}^{3+}$ and $\mathrm{Cl}^{-}$ions. For $\mathrm{CA}_{\text {cluster }}$ fabrication, $\mathrm{CA}$ was firstly dissolved in DMSO at a concentration of $10 \mathrm{~g} / \mathrm{ml}$, and then dropwise added into the Gd-H1/PEG solution in the ultrasonic.

\section{Characterization of physicochemical properties}

The nanocrystal morphology and lattice structure were observed on a high-resolution transmission electron microscope (F20, FEI) operated at $200 \mathrm{kV}$. The hydrodynamic size distribution was obtained from the DLS measurement (Malvern Zetasizer Nano ZS system). UV-vis absorption spectra was obtained from Shimadzu 3000 spectrophotometer.

\section{MC38 and B16F10 tumor models}

All experimental procedures involving animals were conducted in accordance with Institution Guidelines and were approved by the Laboratory Animal Center of Xi'an Jiaotong University. Cancer cells were harvested when they reached near confluence by incubation with $0.05 \%$ trypsinEDTA. Cells were pelleted by centrifugation and resuspended in sterile PBS. MC38 or B16F10 cells $\left(4 \times 10^{6}\right.$ cells/site) were implanted subcutaneously into hip of four- to five-weeks-old male athymic nude mice. When the tumors reached average volume of $\sim 50 \mathrm{~mm}^{3}$, the mice were randomly divided into different groups (five mice per group), and treatment was initiated. Tumor length and width were measured with calipers, and tumor volume was calculated using the following equation: tumor volume $(V)=$ length $\times$ width $^{2} / 2$. For histological examination, the tumor, liver, kidney, heart, spleen and lung tissues were fixed with formaldehyde, dehydrated, sliced into $5.0 \mu \mathrm{m}$ sections and 
subjected to H\&E or immunohistochemical staining. Besides, blood routine examinations (white blood cells, red blood cells and thrombocyte) were carried out at the Clinical Laboratory, the First Affiliated Hospital of Xi'an Jiaotong University, according to the standard clinical laboratory procedures.

\section{Immunohistochemical (IHC) staining}

Sections were cut at $4 \mu \mathrm{m}$ thickness, deparaffinized and rehydrated. Endogenous peroxidase activity was blocked with hydrogen peroxide/methanol, and antigen retrieval was performed in a pH 9.0 TE (Tris-EDTA) buffer by autoclave for $10 \mathrm{~min}$. The resultant tissue sections were then incubated with the antibodies against $\beta$-catenin, Cyclin D1, and c-Myc at $4^{\circ} \mathrm{C}$ overnight. After incubation with labeled streptavidin-biotin (LSAB) complex for $15 \mathrm{~min}$, the slides were stained and visualized by using the iView DAB detection system (ZSGB-BIO, P.R. China). Each stained section was evaluated by a minimum of 10 randomly selected $\times 20$ high-power fields for further statistical analysis.

\section{Double immunofluorescence staining of $\mathrm{CD}^{+} / \mathrm{CD8}^{+} \mathrm{T}$-cells and $\mathrm{CD4}^{+} / \mathrm{CD25}^{+}$}

The primary antibodies comprised a mixture of two antibodies: CD4 [rabbit antibodies]/CD8 [mouse antibodies] or CD4 [rabbit antibodies]/CD25 [mouse antibodies]. For secondary antibodies, the mixture of Alexa Fluor Cy3-conjugated goat-anti-mouse IgG and Alexa Fluor 488-conjugated goat-anti rabbit $\operatorname{IgG}$ were used in this study. Tumor tissues that showed double staining in the immunofluorescence procedure were manually counted in six fields at $400 \times$ original magnification.

\section{Enzyme-Linked Immunosorbent Assay of IFN- $\gamma$ and granzyme}


Enzyme-linked immunosorbent assays (ELISAs) were performed with paired antibody sets, as recommended by the manufacturer (Jianglai Bio, Shanghai, PRC). 
2. Supplementary figures

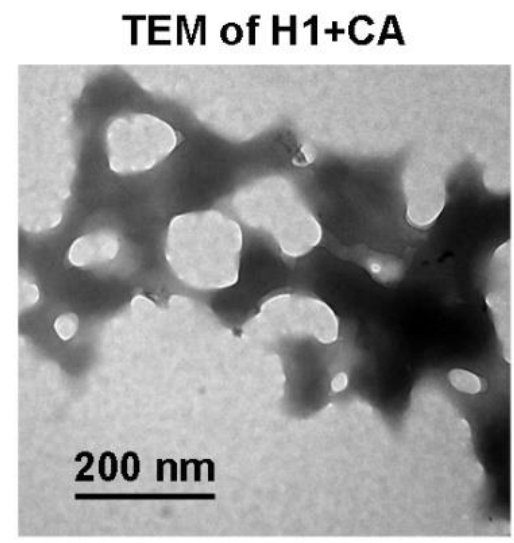

Figure S1. TEM images of the co-assembly of $\mathrm{H} 1$ with CA. 
a

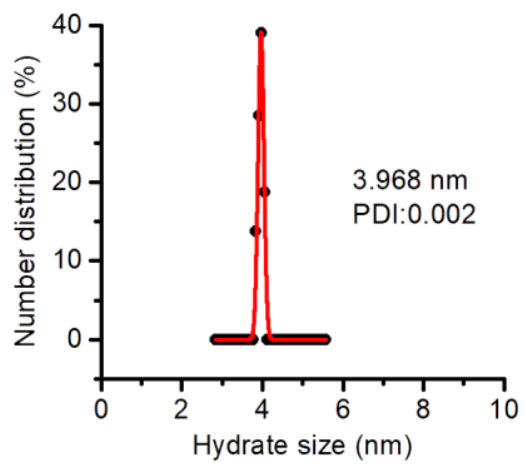

b

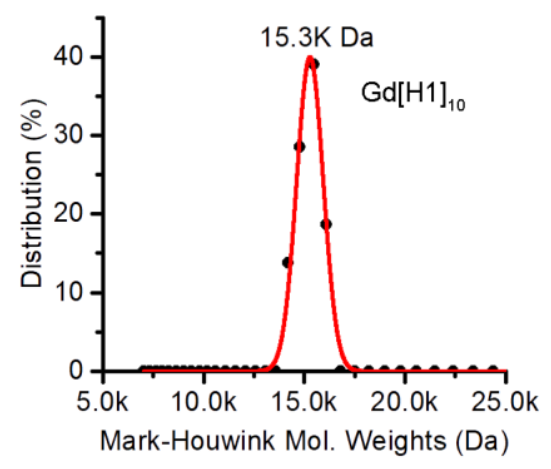

Figure S2. a) Hydrate size of Gd-H1 measured by dynamic light scattering. b) Molecular weight distribution of the Gd-H1 measured by the Mark-Houwink-Sakurada method, which uses empirical constants to calculate the molecular weight from the diffusion coefficient determined from the autocorrelation function of the scattered light. 


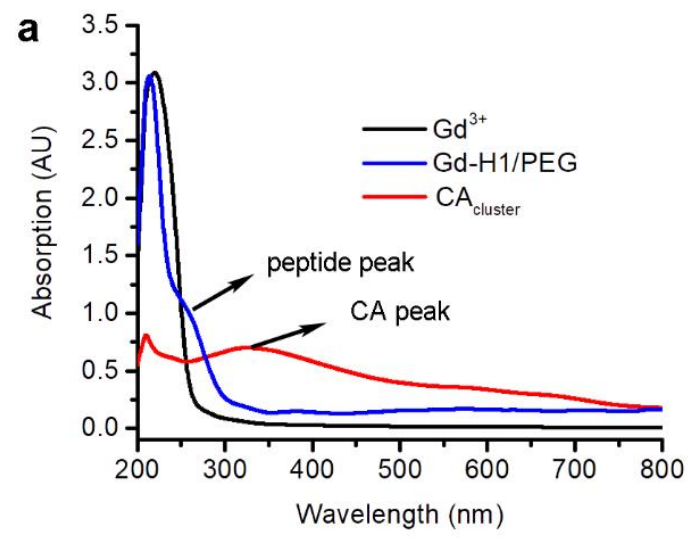

b

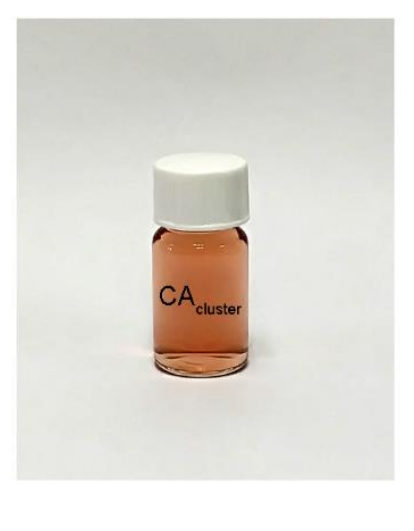

Figure S3. a) Ultraviolet-visible spectra of $\mathrm{Gd}^{3+}$ ions, $\mathrm{Gd}-\mathrm{H} 1 / \mathrm{PEG}$ and $\mathrm{CA}_{\text {cluster }}$ measured in ultrapure water at the concentration of $0.1 \mathrm{mg} / \mathrm{ml}$. b) Solution photos of the $\mathrm{CA}_{\text {cluster }}$ at the concentration of $0.5 \mathrm{mg} / \mathrm{ml}$. 


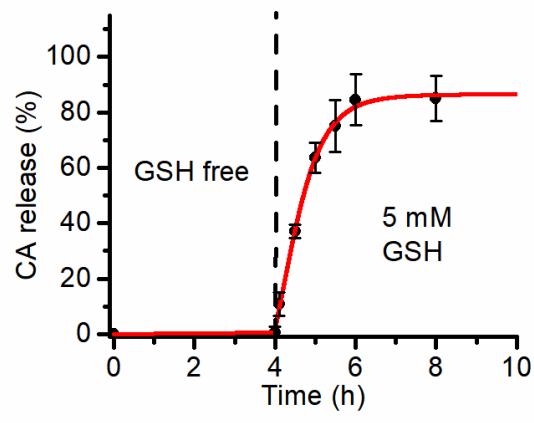

Figure S4. The release kinetics curve of CA in $\mathrm{CA}_{\text {cluster }}$ responding to PBS containing $5 \mathrm{mM} \mathrm{GSH}$. In this test, $0.5 \mathrm{mg} \mathrm{CA}_{\text {cluster }}$ was dissolved in $5 \mathrm{ml}$ PBS (pH 7.4) containing $5 \mathrm{mM} \mathrm{GSH}$. For CA quantification, $100 \mu \mathrm{L}$ mixed liquor were extracted at each time point for $10000 \mathrm{~g}$ centrifugation, and CA in supernatant was quantified by HPLC. 
a

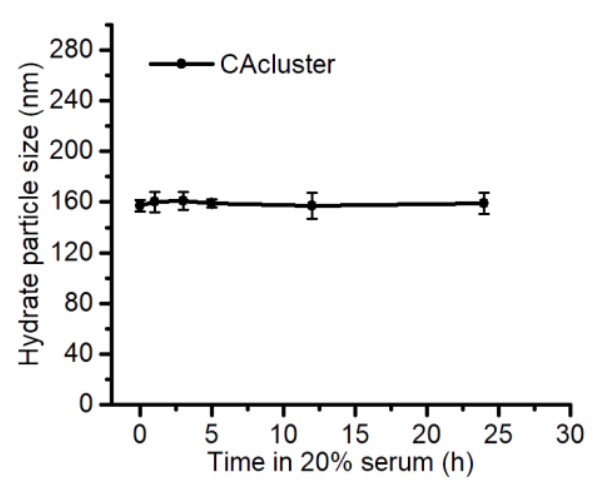

b

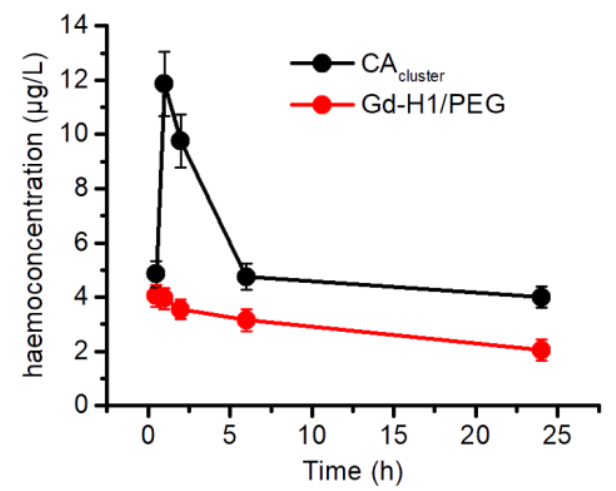

Figure S5 (a) Colloidal stability test of $\mathrm{CA}_{\text {cluster }}$ against 20\% FBS in PBS (pH7.4). Hydrate particle sizes at different time points were measured by DLS at $25^{\circ} \mathrm{C}$. (b) Haemoconcentration of CAcluster and Gd-H1/PEG measured by ICP-MS after intraperitoneal injection at a dose of $1 \mathrm{mg} / \mathrm{Kg}$. 


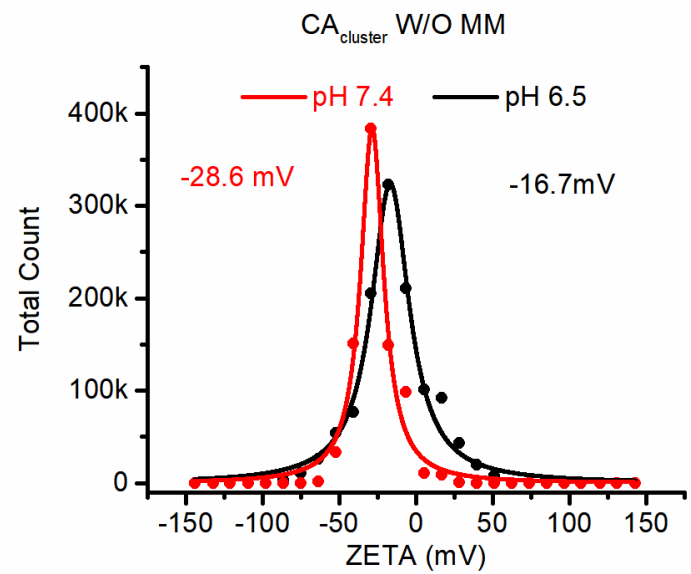

Figure S6. Zeta potential of $\mathrm{CA}_{\text {cluster }}$ without $\mathrm{MM}$ measured at $\mathrm{pH} 6.5$ and $\mathrm{pH}$ 7.4. 
a

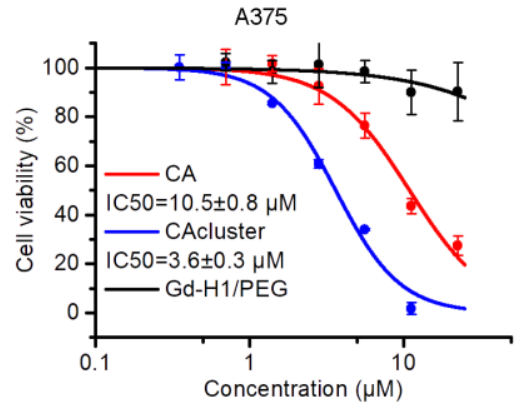

b

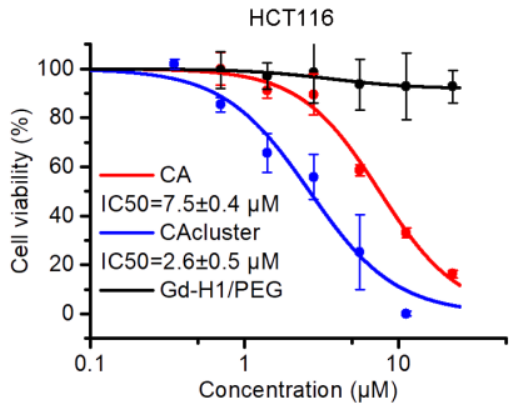

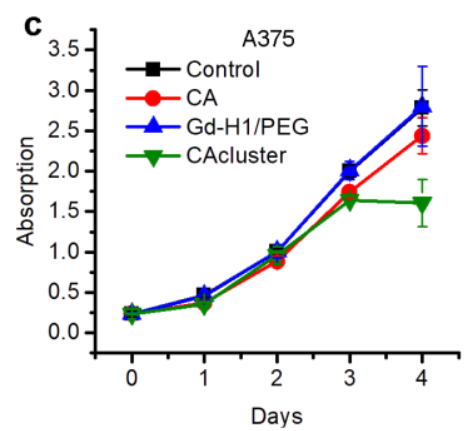

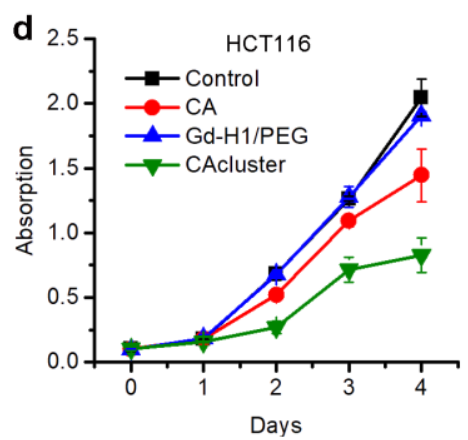

e

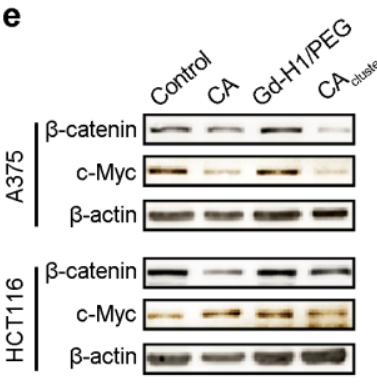

Figure S7. In vitro anti-cancer efficacy. (a and b) Dose-response curves of different nanoparticles formulations against A375 (a) and HCT116 (b) cells after a 72-h incubation with CA, CAcluster and Gd-H1/PEG. (c and d) MTT tetrazolium assay to monitor the effects of CA, CAcluster and GdH1/PEG at the concentration of $10 \mu \mathrm{M}$ on the growth of A375 and HCT116 cells. Each set of data is expressed as mean \pm s.d. $(n=4)$. (e) The result of western blot analysis to monitor the change in protein expression of c-Myc and total $\beta$-catenin in A375 and HCT116 cells treated with $5 \mu \mathrm{M} \mathrm{CA}$, CAcluster and Gd-H1/PEG for $24 \mathrm{~h}$. $\beta$-actin was used as an internal reference. 
a

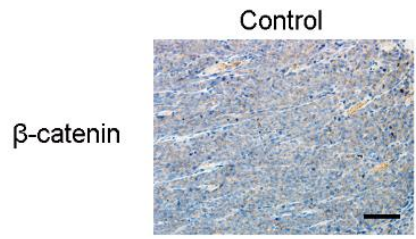

c-Myc

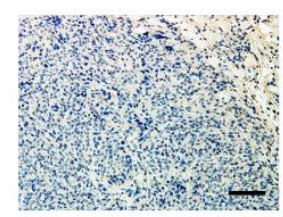

ki67

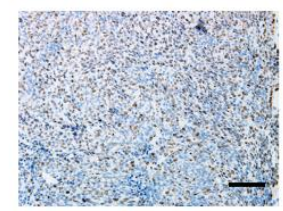

b

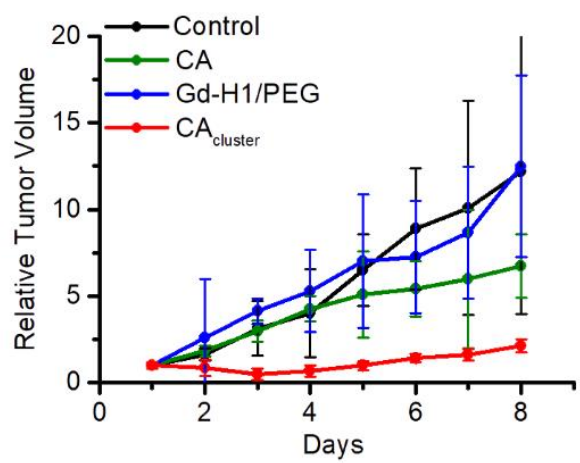

CA
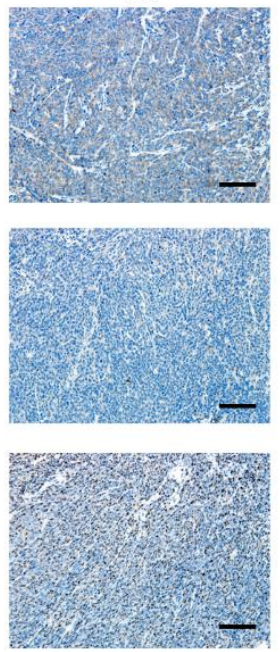

Gd-H1/PEG
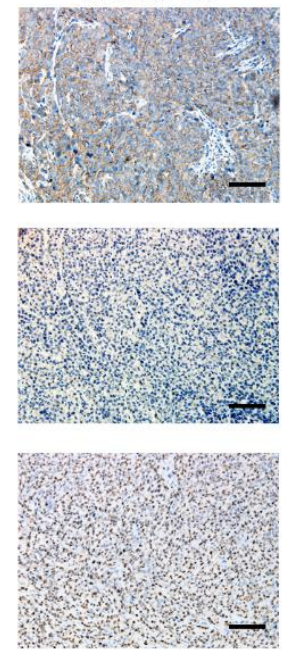

$\mathrm{CA}_{\text {cluster }}$
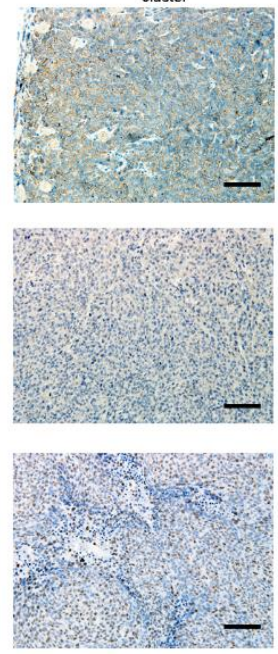

C

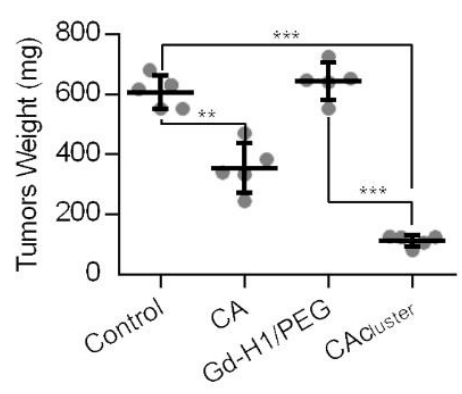

Figure S8. CAcluster potently suppresses Wnt/ $\beta$-catenin signal pathway in HCT116 Xenograft model. (a) The IHC staining of $\beta$-catenin, ki67, and c-Myc in tumor sections from mice with the indicated treatments (scale bar: $100 \mu \mathrm{m}$ ). d). (b) Growth curves of HCT116 xenograft tumors in nude mice with the indicated treatments (mean \pm s.d., $n=5$ ). (c) Tumor weight from mice with the indicated treatments. 


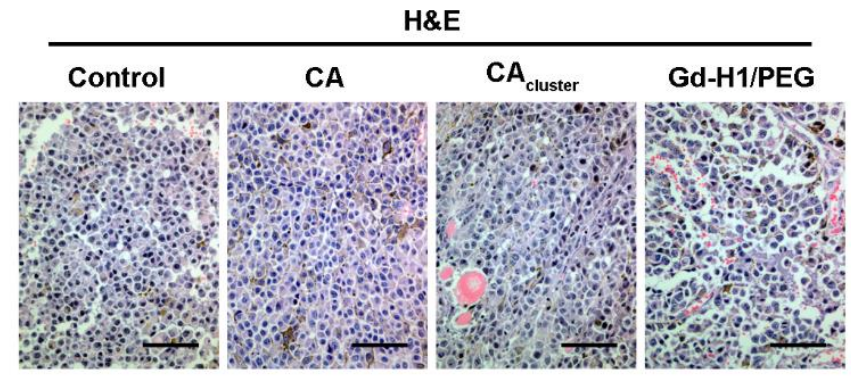

Figure S9. H\&E staining of B16F10 tumors in C57 mice with the indicated treatments. (scale bar: $100 \mu \mathrm{m})$ 


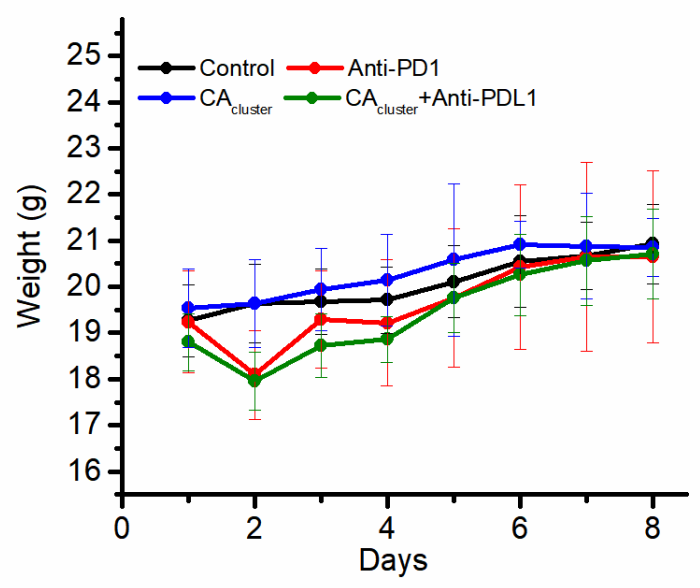

Figure S10. Body weight of mice bearing B16F10 tumors with the indicated treatments. The data were presented as mean \pm s.d. ( $\mathrm{n}=5 /$ group). 


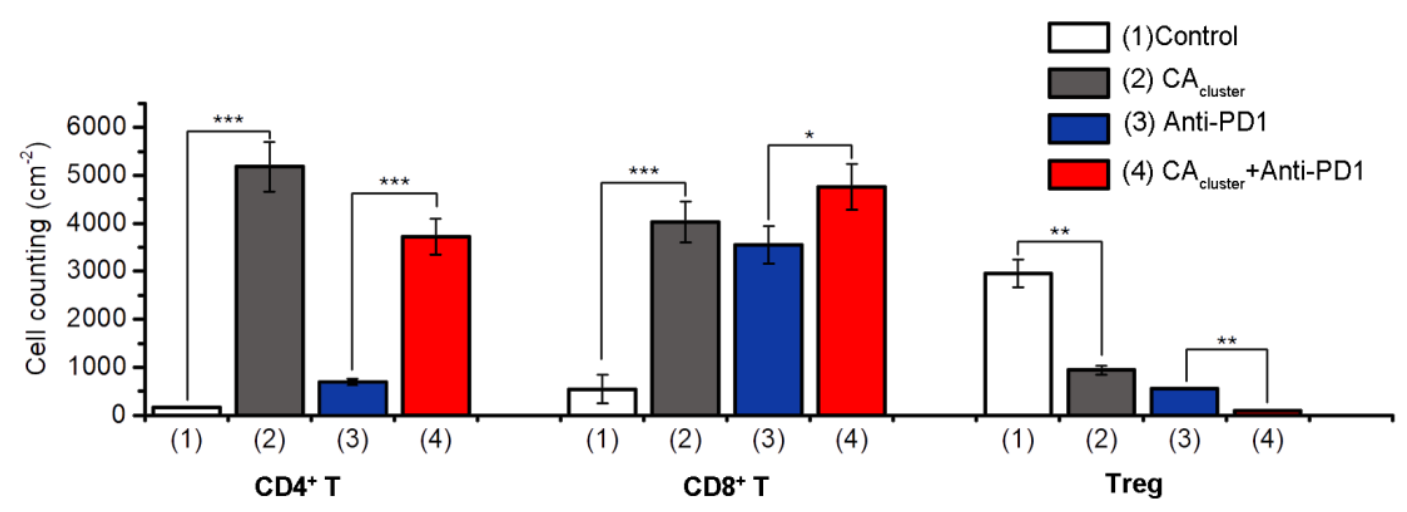

Figure S11. Cell counting from immunofluorescence images of CD3+/CD4+ (Figure5a), $\mathrm{CD} 3+/ \mathrm{CD} 8+$ (Figure5b), and CD4+/CD25+ (Figure5d) cells in tumor sections from mice with the indicated treatments. Sampleswere obtained after two drug injections at the day 4. 


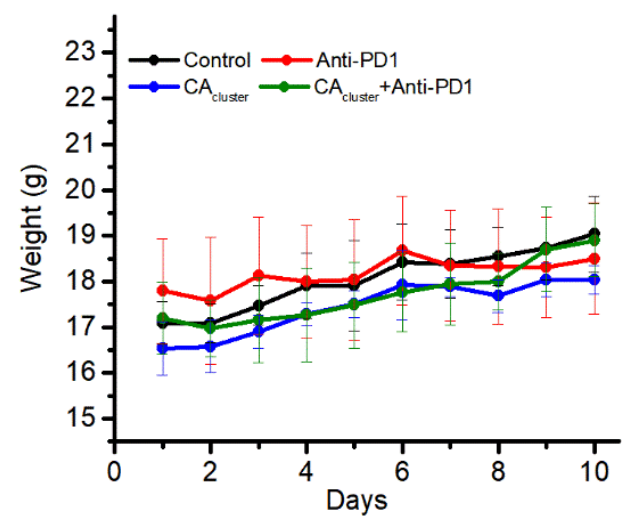

Figure S12. Body weight of mice bearing MC38 tumors with the indicated treatments. The data were presented as mean \pm s.d. ( $n=5 /$ group). 

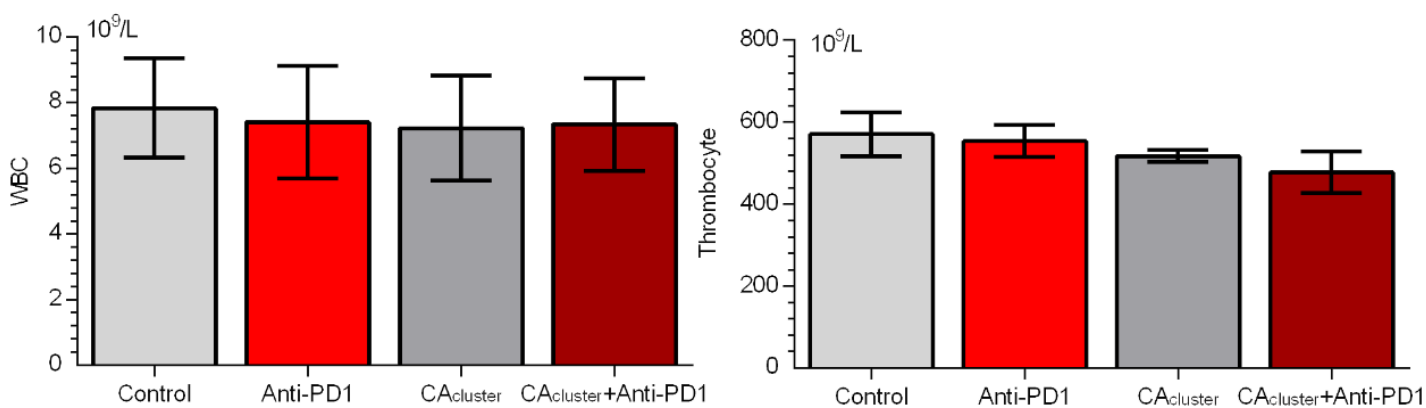

Figure S13. Analysis of white blood cell (WBC) and thrombocyte in mice bearing MC38 tumors after the indicated treatments. The data were presented as mean \pm s.d. ( $\mathrm{n}=5 /$ group). 

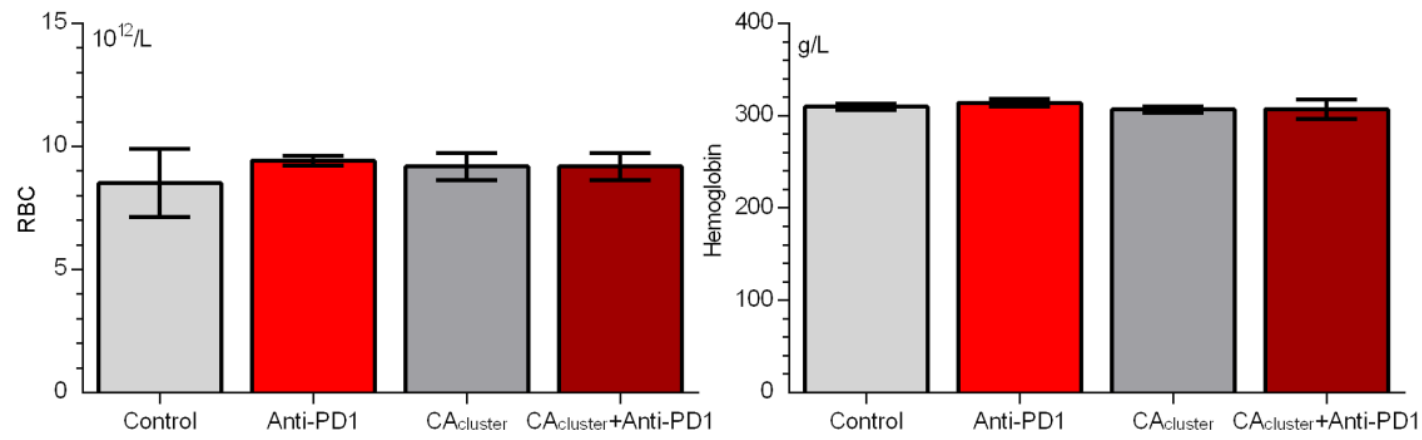

Figure S14. Analysis of red blood cell (RBC) and Hemoglobin in mice bearing MC38 tumors after the indicated treatments. The data were presented as mean \pm s.d. ( $n=5 /$ group). 


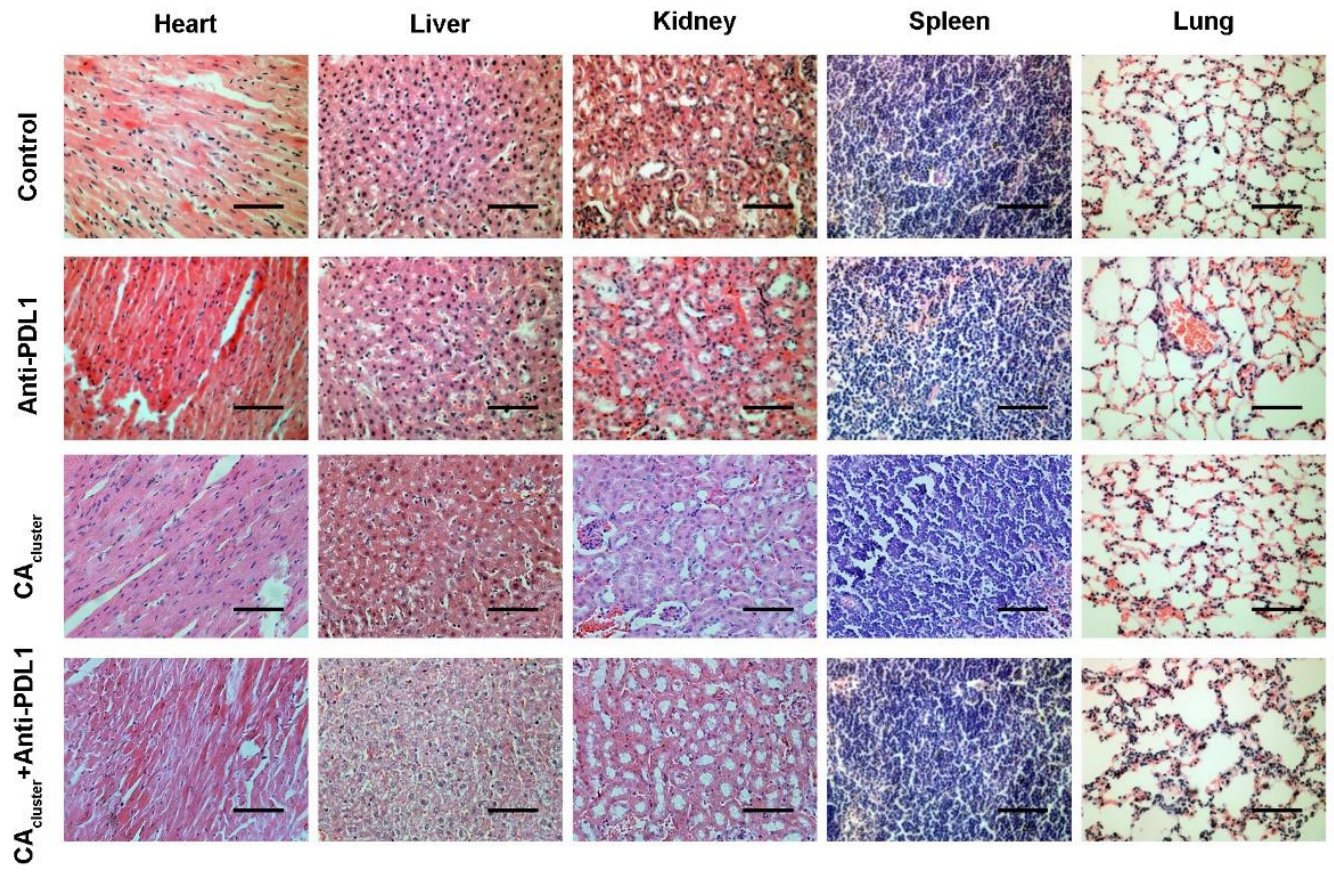

Figure S15. H\&E staining of heart, liver, kidney, spleen and lung tissues in mice bearing MC38 tumors with the indicated treatment (scale bar: $100 \mu \mathrm{m}$ ). 